\title{
ANGULAR MOMENTUM LOSS AND TRANSFER IN CLOSE BINARIES: EFFECTS ON A HUMAN TIME-SCALE?
}

\author{
C. MACERONI \\ Rome Astronomical Observatory, I-00040 Monteporzio C. (RM), Italy; CNRS Institut \\ d'Astrophysique de Paris, France \\ maceroni@coma.mporzio.astro.it
}

The orbital periods of binaries are known to great accuracy, their changes produce an easily detectable cumulative effect and many systems have been observed for more than a century. In tidually locked late-type binaries the orbital period changes are often related to structural or evolutionary changes. The study of the orbital period secular evolution can therefore provide information on phenomena taking place on timescales very short when compared to the typical stellar evolutionary scales, but still much longer thant the human lifetime. This paper focuses on the dynamical evolution due to magnetic braking in late-type close binaries and on the detectability of angular momentum transfer among the stellar layers. 